\begin{tabular}{ccc}
\hline International Journal of Engineering \& Technology, $7(3.1)(2018) 118-120$ \\
SPC \\
Website: www.sciencepubco.com/index.php/IJET \\
Research paper
\end{tabular}

\title{
Farey Sequence for Error Correcting Codes and Medical Images
}

\author{
M Poornima ${ }^{1}$, S Jagannathan ${ }^{2}$, R Chandrasekhar $^{3}$, S T Kumara ${ }^{4}$ \\ ${ }^{I}$ Department of Mathematics, SJB Institute of Technology, Bangalore, India. \\ ${ }^{2}$ Department of Computer Science Engineering, Jain University, Bangalore, India. \\ ${ }^{3}$ Department of MCA, PESIT Research, Bangalore, India. \\ ${ }^{4} A$ P S College of Engineering, Bangalore, 560082, India. \\ *Corresponding author E-mail: poornima581@gmail.com
}

\begin{abstract}
A new scheme for Error Correcting Codes and the coding of medical images through Farey Sequence is brought in the paper. It brings out the reduced real point representations and more of integer point representations. The test case and outcomes are explained in the section 4 and 5.
\end{abstract}

Keywords: Error correcting codes, image coding, reduced arithmetic floating point, fixed point, digital signal processing and farey sequences.

\section{Introduction}

The efficacy of Farey sequence applications for error correcting codes and image processing are brings out in the paper. John Farey came across and observed simple, irreducible and decimal division quantities or fractions which are proper in the interval [0 1] called as Farey sequences. Farey sequences studies and research works carried out are in [1-4].

Implementation of image processing algorithms consists of computer intensive arithmetic operations. Custom digital processing chips convert the floating point computations to fixed point computations and it take more time, space and memory.

This paper in Section 2 gives the properties [3-4] and Section 2 (properties), Section 3 gives Gram-Schmidt orthogonalization, Section 4 gives. Farey Sequence and coding of medical images and also Section 5 gives Farey Sequence and Error Correcting Codes.

\section{Principles and Axioms of the Farey Se- quence}

An Farey Sequences for $F_{1} \ldots F_{8}$ which are irreducible or simple decimal division quantities in $[0,1]$ for a given $\mathrm{n}$ is given below.

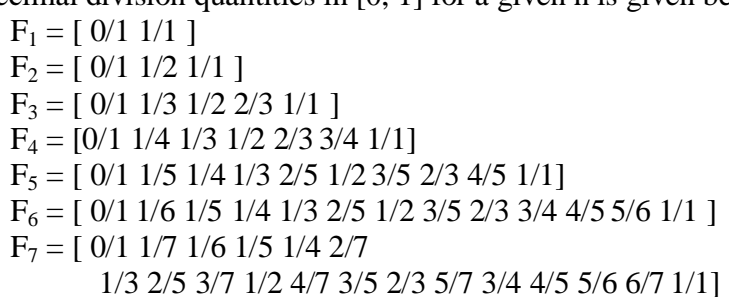

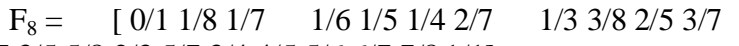
4/7 3/5 5/8 2/3 5/7 3/4 4/5 5/6 6/7 7/8 1/1]

Fig. 1: Examples of Farey Sequences for $F_{1} \ldots F_{8}$

The Principles and axioms of the Farey sequence, the number of decimal division quantities in Farey sequence and the aspects of rank of the division quantities or fractions in a Farey sequence are given in [3 4].

\section{Gram-Schmidt Orthogonalization}

It also called the Gram-Schmidt process, gives an orthogonal basis over a random interval with respect to a weighting function for a non-orthogonal set of linearly independent vectors. A matrix A is set to be orthogonal if its inverse is equal to its transpose $\left(\mathrm{A}^{-1}=\right.$ $\mathrm{A}^{\mathrm{T}}$. The determinant value of any orthogonal matrix $A$ needs to be +1 or -1 . Orthogonal matrices are very much required for proper transmission of binary elements $1111 \ldots 0000$ or vectors.

\section{Farey Sequence and Image Coding of Med- ical Images}

Implementation of image processing algorithms consists of computer intensive arithmetic operations like multiplications and divisions. It needs to be converted to integer or fixed point in order to save time, space and memory. The computation using farey Trees will reduce the floating point arithmetic and reduces to small number of lookups. The performance is much better than the more than known methods mentioned in [5-15].

An image frame of a medical image is considered. All the pixel values are divided by 255 or 65536 depending on the pixel size value for normalization or thresh holding. By normal division it involves more floating arithmetic and computation. It also consumes more power. 
As per the procedure in the Fig 2 the farey approximation can be obtained. The approximation is reduced approximation. The numerator and denominator of the (irreducible) fraction like 159/255 is reduced to $15 / 17$ or $8 / 9$ which is a reduced fraction. Similarly any value like $255 / 65536$ can be reduced to $1 / 257$ reduced fraction. The numerator and denominator of the (irreducible) fraction like $146 / 255$ is reduced to $47 / 82$ or $19 / 37$ which is a reduced fraction. Similarly any value like $246 / 255$ can be reduced to $82 / 85$ reduced fraction.

The Fig 3 give the reduced farey approximation of numerator and denominator of the reduced fraction when pixel value is divided by 255 value.

Further reduced numerator and denominator can be obtained by applying farey approximation as per the Fig $2 \& 3$ repeatedly til the $\operatorname{gcd}($ Numerator, denominator) is 1 . One can stop for particular approximation.

A farey approximation of reduced fraction cab be created for a image of suitable size can be created. By look up for particular fraction, the computation and calculations involving divisions can be reduced.

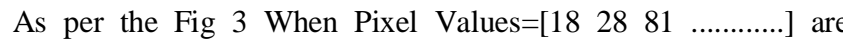
thresholded or divided by 255 will have big real value. But by the farey sequence method will have the reduced fraction consists of Numerator $=\left[\begin{array}{lllll}1 & 1 & 17 \ldots \ldots .\end{array}\right]$ and Denomenator $=\left[\begin{array}{llll}14 & 9 & 22 \ldots . . .\end{array}\right.$ ]. This helps in digital signal processing and fixed point implementation.

\section{Farey Sequence and Error Correcting Codes}

All communications need Error correcting codes which are and are very much important to embedded systems. A fundamental application of coding theory is the detection and correction of errors. R W Hamming and Shannon did much of the early work into error detection and correction for coding theory.

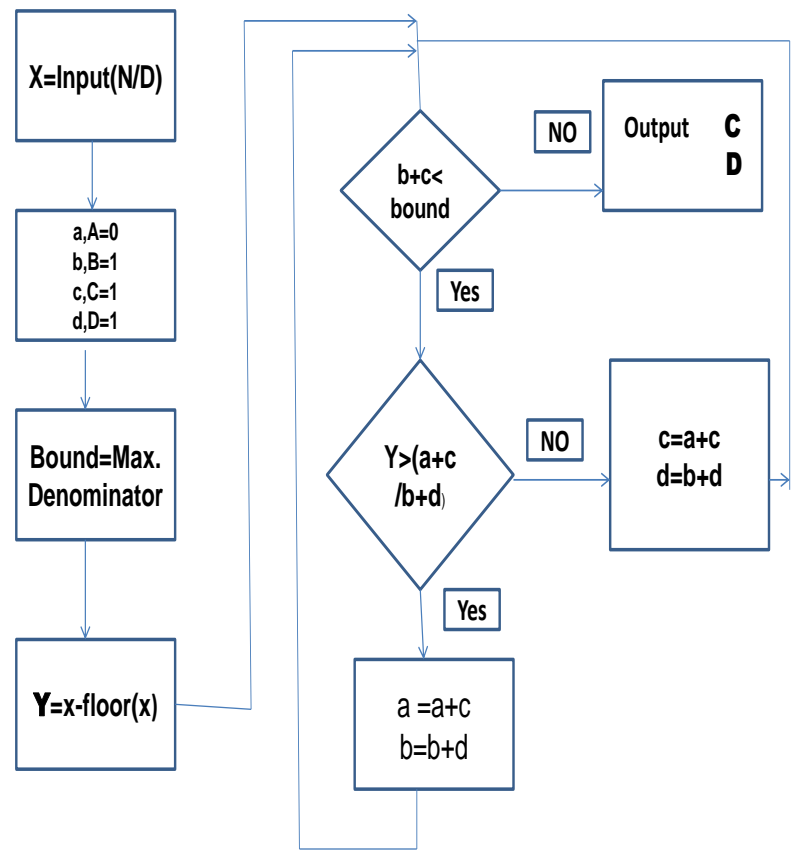

Fig. 2: Procedure for Farey Approximation
By using Farey sequency one can reduce transmission errors. The usefulness of farey sequence for error correction is shown below with typical examples.

$\mathrm{fw}$ is $\mathrm{F}_{7}$ farey sequence matrix.

orfw is orthogonal matrix and orfw' is transpose of the orfw matrix.

Encoding Transmission matrix $=$ orfw $^{*} \mathrm{t} 1$ ' wheren $\mathrm{t} 1$ is test vector Decoding $=$ orfw $* *$ Transmitted Matrix

$\mathrm{F}_{7}=$

$\{0 / 1,1 / 7,1 / 6,1 / 5,1 / 4,2 / 7,1 / 3,2 / 5,3 / 7,1 / 2,4 / 7,3 / 5,2 / 3,5 / 7,3 / 4,4$ $15 \quad 5 / 6 \quad 6 / 7 \quad 1 / 1$

fw $/ *$ ferry Sequence $* /$

$\mathrm{fw}=\mathrm{F}_{7=}$

$\begin{array}{llll}0.1429 & 0.2857 & 0.5000 & 0.7143\end{array}$

$\begin{array}{llll}0.1667 & 0.3333 & 0.5714 & 0.7500\end{array}$

$\begin{array}{llll}0.2000 & 0.4000 & 0.6000 & 0.8000\end{array}$

$\begin{array}{llll}0.2500 & 0.4286 & 0.6667 & 0.8333\end{array}$

>> orfw $\quad / *$ Matrix Fw orthogonalization */

orfw $=$

$\begin{array}{llll}-0.4385 & -0.7006 & 0.0384 & 0.5616\end{array}$

$\begin{array}{llll}-0.4795 & -0.2727 & -0.4807 & -0.6817\end{array}$

$\begin{array}{lllll}-0.5181 & 0.1580 & 0.7987 & -0.2620\end{array}$

$\begin{array}{llll}-0.5562 & 0.6402 & -0.3599 & 0.3889\end{array}$

> orfw' $/ *$ transpose of orthogonalization matrix */

orfw' $=$

$\begin{array}{llll}-0.4385 & -0.4795 & -0.5181 & -0.5562\end{array}$

$\begin{array}{llll}-0.7006 & -0.2727 & 0.1580 & 0.6402\end{array}$

$\begin{array}{llll}0.0384 & -0.4807 & 0.7987 & -0.3599\end{array}$

$\begin{array}{llll}0.5616 & -0.6817 & -0.2620 & 0.3889\end{array}$

>> s1 /* Test vextor*/

$\mathrm{s} 1=$

$\begin{array}{llll}1 & 2 & 1 & 1\end{array}$

> s2 /* Test vextor*/

$s 2=$

$\begin{array}{llll}1 & 0 & 1 & 0\end{array}$

> test1

test $1=$ orfw $*$ test 1 ' (Encoding)

$-1.2397$

$-2.1872$

0.3346

0.7532

> trtest1 /*Transmission*/

Transmitted with error in element 1(-1.2387 instead of -1.2397 , element $2(-2.1873$ instead of -2.1872 , element $3(0.3342$ instead of $0.3342)$ and element $4(0.7531$ instead of 0.7532$)$

trtest $1=$

$-1.2387$

$-2.1873$

0.3342

0.7531

> dtest $1 / *$ Decoded $* /$

dtest $1=$ orfw $*$ trtest 1

0.9999

1.9992

0.9998

1.0007

Decodes as [ $\left[\begin{array}{llll}1 & 2 & 1 & 1\end{array}\right]$

> test2

test $2==$ orfw*test2' (Encoding)

$-0.4001$

$-0.9602$

0.2806

$-0.9161$

> trtest 2 /*Transmission*/

Transmitted with error in element 4(--0.9159 instead of -0.9161) trtest $2=$

$-0.4001$

$-0.9602$

0.2806

$-0.9159$ 


\section{$>$ dtest $2 \quad / *$ Decoded $* /$ \\ dtest $2=$ orfw $*$ trtest 2 \\ 0.0381 \\ $-0.4822$ \\ 1.1586 \\ $-0.6509$}

Decoded as 10010

$>$

Everey error prone digit in the transmitted digits like element 1(1.2387 instead of -1.2397 , element $2(-2.1873$ instead of -2.1872 , element 3(0.3342 instead of 0.3342) and element 4(0.7531 instead of 0.7532 ) are decoded correctly, and finally round the value to get the correct value.

Farey tables for transmission and decoding can be used for fast processing. Farey sequence can be used for real and integer data for error correcting data.

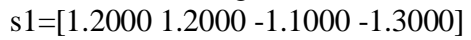

test $1=$ orfw $^{*} \mathrm{~s} 1(1,1: 4)$,

test $1=$

$-2.1392$

0.5123

$-0.9701$

$-0.0090$

Decoded Dtest $1=$ orfw ${ }^{*} *$ test 1 (Encoding)

[1.2000 1.2000 -1.1000 -1.3000]

By corrupting test 1 in the element 1 and transmitting as

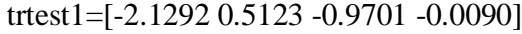

Decoding

Dtest $1=$ orfw $*$ trtest 1

Dtest $1=[1.19561 .1930-1.0996-1.2944]$

Rounding yields

[1.2000 1.2000 -1.1000 -1.3000]

Which is same as $\mathrm{s} 1$

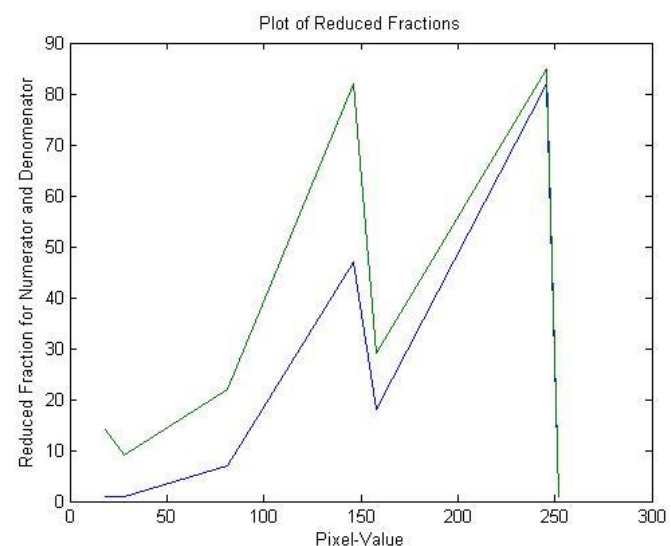

Fig. 3: Pixel Value and Further Reduced Fraction

\section{Conclusion}

This paper shows adaptation of farey sequences for error correcting codes and obtaining a decimal fraction in the Farey sequence. Handling of the fractions can been improved by using the Farey approximation approach and proposed variable processing architecture. New scheme to obtain a decimal division quantity closest to any random decimal division quantities in a given Farey sequence are also explained in the paper. Most of the operations are reduced real point representations and also are of reduced time, space and memory.

\section{References}

[1] R. Graham etc., Addison-Wesley, 1994. 2. C. E. Patrascu and M.Patrascu. Computing Order Statistics in the Farey Sequence, issue ANTS 2004: 358-366

[2] J. Pawlewicz, Order statistics in the Farey sequences in sublinear time and counting primitive lattice points in polygons. Algorithmica 55(2): 271-282, 2009
[3] S. Das, K. Halder, S. Pratihar, and P. Bhowmick, Properties of Farey Sequence and their Applications to Digital Image Processing, 4th Intl. Conf. on Information Processing, 6-8 August, Bangalore University, Bangalore, pp. 71-81, 2010 (IK International Publishing House Pvt. Ltd. New Delhi, 2010, ISBN 978-93-80578-46-0).

[4] Jonathan Ainsworth, etc., The Farey Sequence, School of Mathematics, University of Edinburgh, March 15, 2012.

[5] G. H. Hardy and E. M. Wright. An Introduction to the Theory of Numbers. OxfordUniversity Press, New York, 1968

[6] E. H. Neville. The Farey Series of Order 1025. Cambridge University Press, 1950.

[7] P. L. Rosin. Techniques for assessing polygonal approximation of curves. IEEE Trans.PAMI, 19(6): 659-666, 1997

[8] M. Schroeder. Fractions: Continued, Egyptian and Farey (Chapter 5). Number Theory inSc. \& Communication, vol. 7, Springer Series in Information Sciences, 2006.

[9] C.-H. Teh and R. T. Chin. On the detection of dominant points on digital curves. IEEE Trans. PAMI, 2(8):859-872, 1989.

[10] P. Y. Yin. Ant colony search algorithms for optimal polygonal approximation of planecurves. Pattern Recognition, 36:1783-1797, 2003.

[11] K. Wall and P.-E. Danielsson, A fast sequential method for polygonal approximation of digitized curves, Computer Vision, Graphics, and Image Processing, 28:220-227, 1984.

[12] Narkhede H P , Review of Image Segmentation Techniques, International Journal of Science and Modern Engineering (IJISME) ISSN: 2319-6386, Volume-1, Issue-8, July 201354.

[13] Digital Image Processing, Rafael C. Gonzalez \& Richard E. Woods, Second Edition 2002, Prentice Hall.

[14] K. K Singh, A Singh, “A Study of Image Segmentation Algorithms for Different Types of Images", International Journal of Computer Science Issues, Vol. 7, Issue 5, 2010.

[15] Chunming Li, Rui Huang, Zhaohua Ding, J. Chris Gatenby, Dimitris N. Metaxas, A Level Set Method for Image Segmentation, in the Presence of Intensity nhomogeneities, With Application to MRI, IEEE Transactions on Image processing, Vol. 20, NO. 7, July 2011. 